\title{
Fast recursive computation of Krawtchouk polynomials
}

\begin{abstract}
Krawtchouk polynomials (KPs) and their moments are used widely in the field of signal processing for their superior discriminatory properties. This study proposes a new fast recursive algorithm to compute Krawtchouk polynomial coefficients (KPCs). This algorithm is based on the symmetry property of KPCs along the primary and secondary diagonals of the polynomial array. The $n-x$ plane of the KP array is partitioned into four triangles, which are symmetrical across the primary and secondary diagonals. The proposed algorithm computes the KPCs for only one triangle (partition), while the coefficients of the other three triangles (partitions) can be computed using the derived symmetry properties of the KP. Therefore, only N / 4 recursion times are required. The proposed algorithm can also be used to compute polynomial coefficients for different values of the parameter $p$ in interval $(0,1)$. The performance of the proposed algorithm is compared with that in previous literature in terms of image reconstruction error, polynomial size, and computation cost. Moreover, the proposed algorithm is applied in a face recognition system to determine the impact of parameter $\mathrm{p}$ on feature extraction ability. Simulation results show that the proposed algorithm has a remarkable advantage over other existing algorithms for a wide range of parameters $\mathrm{p}$ and polynomial size $\mathrm{N}$, especially in reducing the computation time and the number of operations utilized.
\end{abstract}

Keyword: Krawtchouk polynomial; Krawtchouk moments; Orthogonal polynomials; Signal processing; Propagation error 